\title{
Kaposi sarcoma-associated herpesvirus: immunobiology, oncogenesis, and therapy
}

\author{
Dirk P. Dittmer and Blossom Damania
}

Lineberger Comprehensive Cancer Center Program in Clobal Oncology, Department of Microbiology and Immunology, Center for AIDS Research, School of Medicine, University of North Carolina at Chapel Hill, Chapel Hill, North Carolina, USA.

\begin{abstract}
Kaposi sarcoma-associated herpesvirus (KSHV), also known as human herpesvirus 8, is the etiologic agent underlying Kaposi sarcoma, primary effusion lymphoma, and multicentric Castleman's disease. This human gammaherpesvirus was discovered in 1994 by Drs. Yuan Chang and Patrick Moore. Today, there are over five thousand publications on KSHV and its associated malignancies. In this article, we review recent and ongoing developments in the KSHV field, including molecular mechanisms of KSHV pathogenesis, clinical aspects of KSHV-associated diseases, and current treatments for cancers associated with this virus.
\end{abstract}

\section{Introduction}

Kaposi sarcoma (KS) is the most common cancer in individuals living with HIV/AIDS today $(1,2)$. While the introduction of effective HIV therapy was concurrent with a decline in the incidence of KS in the United States, KS incidence has stabilized and remained essentially level since 2000. In Africa, where KSHV and HIV infections are highly prevalent, $\mathrm{KS}$ is among the most common cancer type in men overall. In some sub-Saharan countries, KS is more prevalent than prostate cancer is in the US (3). As the expected lifespan of individuals living with HIV/AIDS increases, we foresee an increase in all cancers in this population, including KS.

Kaposi sarcoma-associated herpesvirus (KSHV) is necessary for KS development. KSHV DNA is found in all KS lesions $(4,5)$. KS prevalence follows KSHV seroprevalence, and in most cases fulminant KS is accompanied and preceded by a rise in KSHV viral load in blood. In addition to KS, KSHV is also the etiologic agent of the plasmablastic variant of multicentric Castleman's disease (MCD) (6) and primary effusion lymphoma (PEL) $(7,8)$. Moreover, KSHV is the causative agent of KS-immune reconstitution syndrome (KS-IRIS) (9, 10) and KSHV-inflammatory cytokine syndrome (KICS) (11). However, not all KSHV infections lead to KSHV-associated conditions. The majority of primary KSHV infections have no clinical symptoms and, as with other human oncogenic viruses, cancer emerges only after decades of dormancy. KSHV can be transmitted via asymptomatic oral shedding as well as through bodily fluids (12-14). KSHV can infect many different types of cells including endothelial cells, B lymphocytes, monocytes, dendritic cells (DCs), and epithelial cells. KSHV provides a growth advantage to infected endothelial cells. The virus consistently immortalizes, but rarely transforms, primary cells in culture (15-19). It is only under special circumstances and perhaps upon infection of rare progenitor cells with stem cell properties that the interplay between virus and host leads to a fully transformed state.

Conflict of interest: The authors have declared that no conflict of interest exists. Reference information: J Clin Invest. 2016;126(9):3165-3175. doi:10.1172/JCI84418.
Why is the human immune system so powerful in suppressing disease, yet can never eliminate this pathogen? Like all herpesviruses, KSHV establishes lifelong infection in the host and molecular latency in cells in culture. KS is primarily the consequence of systemic viral reactivation from a latent reservoir, most likely a lymph node-resident B cell (20-23). Prior to the emergence of HIV, endemic KS in sub-Saharan Africa was a disease of both children and adults, and classic KS was a disease of elderly men in the Mediterranean region. Today, KS also develops with higher frequency in HIV-infected individuals (HIV-associated KS) compared with HIV-negative individuals, as well as in solid organ transplant recipients (transplant KS). Thus, it appears that KS develops in response to severe T cell depletion or inactivation. Infant, aging-, chemical-, or HIV-induced immune deficiency is an essential cofactor for the development of KS.

\section{Primary infection and the innate immune response to KSHV}

KSHV is thought to enter cells predominantly through the endocytic pathway. Viral attachment involves several different receptor binding proteins on the virion. KSHV can infect multiple cell types, including B cells, endothelial cells, monocytes, and DCs, and hence uses multiple viral receptors to enter the host cell. One such receptor is the $\mathrm{gB}$ glycoprotein, which contains an integrin-binding RGD (Arg-Gly-Asp) motif that plays a role in virion binding and entry of endothelial cells (24-26). Activated B cells, macrophages, and DCs express a DC-specific ICAM-3-grabbing non-integrin (DC-SIGN; CD209) that facilitates KSHV infection in these cell types $(27,28)$. The cysteine transporter $(\mathrm{xCT})$ can also serve as a receptor for the virus (29).

$\mathrm{KSHV}$ is thought to enter cells predominantly through the endocytic pathway (30-32). During its entry into the host cell, the virus encounters multiple innate immune sensors that activate an antiviral response. It is likely that the activation of such innate immune responses during primary infection induces the virus to enter molecular latency, which is a more quiescent and less immunogenic phase of the lifecycle. 


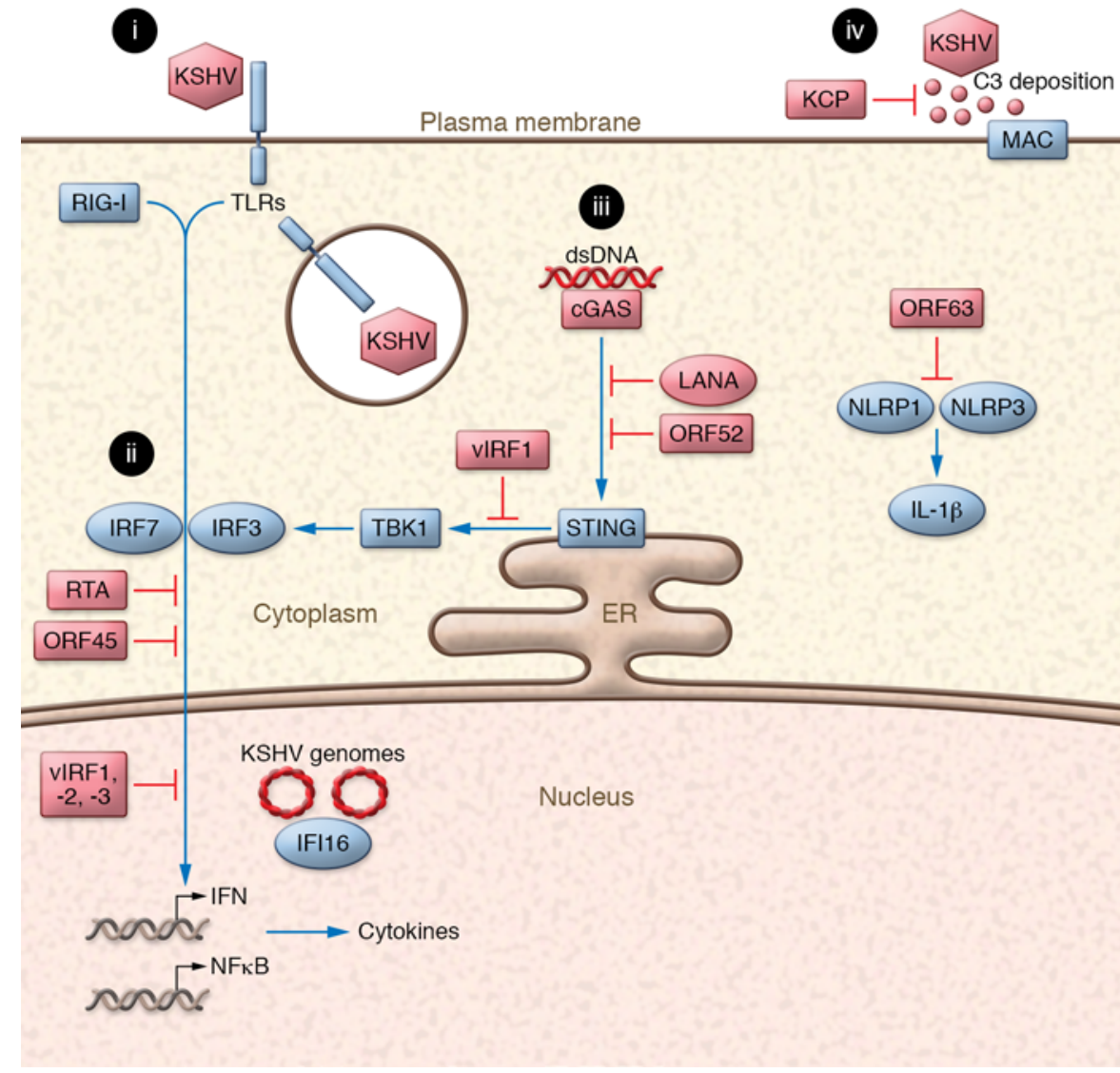

Figure 1. Innate immune evasion by KSHV. KSHV encodes multiple viral proteins that inhibit innate immune pathways. (i) KSHV-mediated activation of TLRs and RIG-I triggers interferon and IFN- $\beta$ production following primary infection. (ii) KSHV Rta, ORF45, and vIRF1, -2 , and -3 block cellular IRFs from activating interferon-responsive genes. (iii) KSHV LANA, ORF52, and vIRF1 block the CGASSTING DNA-sensing pathway. (iv) KSHV ORF63 inhibits NLRP1 and NLRP3 inflammasome activation and KCP/ORF4 promotes KSHV pathogenesis by helping the virus to evade complement.

production and suppresses viral gene expression following de novo infection with KSHV as well as during viral reactivation $(39,40)$.

NLRs. NLR family members can form inflammasomes, a complex comprised of an NLR protein, ASC, and pro-caspase-1. NLRs sense PAMPs, and activation of the NLR inflammasomes results in cleavage and production of active IL- $1 \beta$ and IL-18, which are proinflammatory cytokines. Primary infection with KSHV activates NLRP1 and NLRP3 $(41,42)$ and potentially other NLRs.

ALRs. Like NLRs, ALR family members can also form inflammasomes to activate proinflammatory cytokine signaling. Primary infection with KSHV has been shown to activate the ALR family member interferon gamma-inducible protein 16 (IFI16) $(43,44)$. It was additionally reported that IFI16 can detect KSHV in latently infected cells (43).

cGAS-STING. Cyclic GMP-AMP (cGAMP) synthase (cGAS) and STING are members of the cytosolic DNA-sensing pathway. This cGAS-STING pathway appears to sense KSHV during both primary infection and reactivation from latency in multiple cell types (45-47).

There seems to exist a delicate equilibrium between the virus and host response to infection. Although innate immune activation might help KSHV enter a latent, quiescent phase inside the infected cell and induce expansion of latently infected cells, a high degree of innate immune response

Cells infected with viruses such as KSHV trigger an innate immune response through pattern recognition receptors (PRRs) that recognize pathogen-associated molecular patterns (PAMPs) and lead to the production of interferon and proinflammatory cytokines. It is important to note that each cell type expresses its own unique set of PRRs. There are many different PRRs including TLRs, retinoic acid-like receptors (RLRs), NLRs, absent in melanoma 2 (AIM2)-like receptors (ALRs), and cytosolic DNA sensors (reviewed in ref. 33). Members of the NLR, ALR, and RLR families can form inflammasomes that, upon activation, lead to the production of IL-1 $\beta$ and IL-18 (34). KSHV infection and/or reactivation activates a multitude of PRRs in different cell types; these are described below.

TLRs. Following primary infection, KSHV has been shown to activate the RNA sensor TLR3 in monocytes, (35) and the DNA sensor TLR9 in plasmacytoid DCs (pDCs) (36). Activation of either TLR results in interferon production and upregulation of cytokines and chemokines. KSHV also activates TLR4 signaling that likely occurs through recognition of the viral glycoproteins $\mathrm{gB}$ and K8.1 (37). Stimulation of TLR7/8 in PEL cells has been shown to lead to reactivation from latency (38).

RLRs. The cytosolic RNA sensor RIG-I and its adaptor protein, mitochondrial antiviral signaling protein (MAVS), induces IFN- $\beta$ facilitates killing of the infected cell and ultimately prevents the establishment of latency. To counter the host response to viral infection and reactivation, KSHV encodes many viral genes that blunt innate immune signaling pathways. Some of these viral products are summarized in Figure 1 and are described below.

Viral interferon regulatory factors. KSHV encodes four viral interferon regulatory factors (vIRFs), three of which ablate cellular IRF signaling and inhibit the production of type I IFNs, including IFN- $\alpha$ and IFN- $\beta$ (reviewed in ref. 48). KSHV vIRFs have been shown to inhibit IFN production that lies downstream of TLR3 activation (49), MAVS activation (50), and cGAS-STING activation (45). KSHV vIRF1 also inhibits the function of IFN-induced genes such as ISG15 (51) and the transcription of TLR4 (37).

Complement regulatory proteins. KSHV encodes the complement regulatory protein KCP, which is encoded by ORF4. KCP is part of the virion and functions as a cofactor for factor I-mediated cleavage of $\mathrm{C} 3 \mathrm{~b}$ and $\mathrm{C} 4 \mathrm{~b}$, the complement system's opsonizing factors $(52,53)$. KSHV has also been reported to exploit the host complement system to promote viral persistent infection (54).

Tegument proteins. Tegument proteins are a characteristic feature of all herpesviruses, and a large number of them are deposited into the cytoplasm following virion fusion and capsid release. 
KSHV ORF45 is a tegument protein that blocks IRF7 phosphorylation and activation of type I IFN responses $(55,56)$. ORF64 is another conserved herpesviral tegument protein that encodes potent deubiquitinating activity (57). ORF64 can reduce TRIM25dependent ubiquitination and activation of RIG-I, thereby inhibiting this sensing pathway in KSHV-infected cells (40).

DNA-binding proteins. Although it is primarily a DNA-binding protein and transcription factor, KSHV Rta/ORF50 can also induce the degradation of innate immune sensors such as IRF7, TLR3, and myeloid differentiation factor 88 (MyD88) (58-61). The latencyassociated nuclear antigen (LANA) is another DNA-binding protein that inhibits IFN- $\beta$ induction (62) and the transcription of IFN- $\gamma$ inducible genes (63). Recently, it was shown that cytoplasmic variants of LANA can inhibit the cGAS-STING DNA-sensing pathway by directly binding to cGAS (47). Interestingly, another KSHV open reading frame, ORF52, was similarly shown to bind and inhibit cGAS enzymatic activity. Infection with an ORF52-deficient virus in endothelial cells resulted in increased cGAS signaling (46).

Furthermore, NLRP1 and NLRP3 inflammasome activation is inhibited by the tegument protein KSHV ORF63 during de novo infection, resulting in reduced IL- $1 \beta$ and IL-18 production. ORF63 binds to NLRP1 and interferes with the interaction between NLRP1 and pro-caspase-1 (42).

In addition to the innate immune responses described above, adaptive immune responses also play an important role in KSHV pathogenesis. KSHV expresses proteins that affect antigen presentation, B cell targeting, MHC class I display, and neutrophil and basophil activation. The KSHV-infected cell presents antigenic peptides from the virus in complex with MHC class I to cytotoxic T lymphocytes (CTLs) (64-66). Additionally, KSHV-infected $B$ cells stimulate activation-induced cytidine deaminase (AID) expression and are targeted for elimination by NK cells through upregulation of NKG2D ligands (67). KSHV also encodes genes that inhibit these immune responses. KSHV K3/MIR1 and K5/ MIR2 are ubiquitin ligases that inhibit MHC class I display (68, 69). K3/MIR 1 downregulates four HLA allotypes (HLA-A, B, C, and E), while K5/MIR2 downregulates HLA-A and HLA-B (70, 71). $\mathrm{K} 3$ and $\mathrm{K} 5$ can also downregulate CD1d (72) and IFN- $\gamma$ receptor 1 (IFNGR1) (73). K5 hinders expression of ICAM-1 and the costimulatory molecule $\mathrm{B} 7-2$ (CD86) $(74,75)$. It also downregulates the NKG2D ligands, MHC class I-related chain A (MICA), MICB, and the NKp80 ligand, activation-induced C-type lectin (AICL) (76). KSHV vCD200, also known as viral OX2, is a homolog of cellular CD200 that is broadly expressed and suppresses neutrophil and basophil activation (77) as well as activation of macrophages (78). vCD200 can also function as a negative regulator of antigen-specific $\mathrm{T}$ cell responses, including inhibition of IFN- $\gamma$ production and CD107a mobilization (79).

KSHV viral IL-6 (vIL-6) has also been reported to block interferon signaling. IFN- $\alpha$ directly activates viral IL- 6 gene expression through IFN-inducible sites in the vIL- 6 promoter. vIL- 6 then subsequently blocks IFN signaling by inhibiting IFN induction of p21 and also downregulating the IFN receptor-mediated phosphorylation of TYK2 kinase, thereby dampening JAK-STAT signaling (80).

Finally, KSHV encodes several viral macrophage inflammatory proteins (vMIPs). KSHV vMIP-II inhibits chemotaxis and recruitment of monocytes (81) as well as NK cells (82). KSHV
vMIP-II is an agonist for CCR8, a chemokine receptor that is preferentially expressed on polarized Th2 T cells (83). Similarly, KSHV vMIP-III serves as an agonist for the cellular chemokine receptor CCR4, which is also expressed by Th2 T cells (84). Hence, the KSHV vMIPs skew T cell responses towards a Th2type lymphocytic response, and this may play a role in subverting the host immune response.

\section{Latent KSHV infection and reactivation}

KSHV successfully subverts the cellular innate immune response to establish a lifelong latent reservoir in the infected host, primarily in B cells. The virus has evolved a number of mechanisms to ensure that virally infected B cells outcompete their uninfected counterparts, which in the extreme leads to B cell hyperplasia, such as $\operatorname{MCD}(6,21)$, or B cell neoplasia, such as PEL (7). These include inhibiting apoptosis, overcoming G1 phase arrest, lowering the threshold for B cell receptor (BCR) activation, and providing ligand-independent progrowth signals. In addition to B cells, this virus can also enter CD34 cells, T cells, monocytes, and pDCs $(36,85-88)$, though it is unclear if these cell types contribute to systemic persistence or serve as sentinels to detect infection. EpsteinBarr virus (EBV) also uses B cells as the predominant latent reservoir, as does murine herpesvirus 68 (reviewed in ref. 89); however, there are important biological differences between latent infection in B cells in KSHV and other herpesviruses. EBV is easily detected in blood in circulating $\mathrm{CD}_{3} 8^{+}$memory B cells, which typically emerge from the germinal center. In contrast, KSHV is not readily detectable in circulating B cells (23), and KSHV viral loads in blood are 10- to 100-fold lower than those of EBV or human CMV. These observations suggest that tissue-resident B cells are the predominant latent reservoir for KSHV.

The deepest insights about the biology of KSHV prior to disease come from studying the related murine gammaherpesvirus 68 (MHV-68) and from genetically engineered mouse models. In mouse models, it was possible to define B cell tropism through functional phenotypes (21). Studies in MHV-68 defined the distinction between establishment of latency, which drives the size of the latent reservoir, and persistence of the latent reservoir, i.e., long-term survival of infected cells that are still capable of reactivation (for recent examples see refs. 90-92). We do not know the dynamics of latently infected cells in humans and have only just begun to decipher the physiological signals that modulate reactivation events and thereby transmission and disease in patients.

Histone deacetylation reactivates KSHV. Vorinostat (also known as SAHA) and valproic acid induce reactivation in culture and in patients (93-95). Sodium butyrate and phorbol esters reactivate KSHV from PEL, though only a fraction of episomes is competent for reactivation at any given time $(96,97)$. The majority of the KSHV episome is methylated, transcriptionally silent, and decorated with histone markers, indicative of inactive chromatin (96, 98-101). These markers of methylation are established early in infection and maintained by cellular chromatin remodelers, and organized by CCCTC-binding factor (a zinc finger protein, also known as CTCF) recognition elements (100). During latency, the virus actively engages host chromatin modulators (Figure 2). For instance, LANA binds to histones $\mathrm{H} 2 \mathrm{~A}$ and $\mathrm{H} 2 \mathrm{~B}$ as well as MECP2, and to the BET family proteins BRD2 and BRD3 (102-104). 


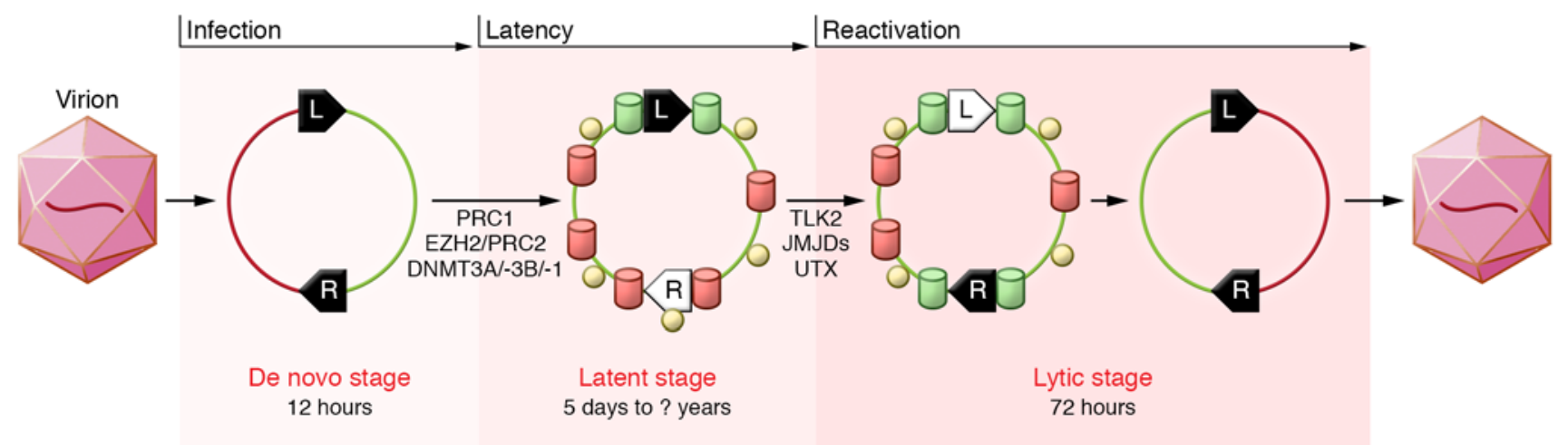

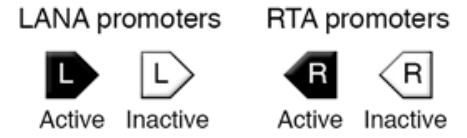

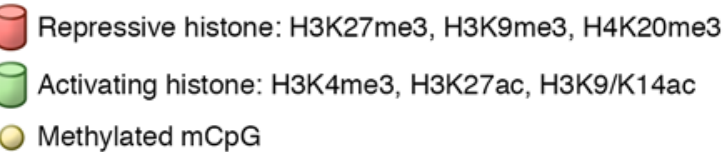

Repressive histone: H3K27me3, H3K9me3, H4K2Ome3

Methylated $\mathrm{mCpG}$

Figure 2. Epigenetic modifications to KSHV upon primary infection, latency, and reactivation. DNA is shown as a single line (linear inside virion, circular as episomal plasmid). Depicted in the figure is the transition of primary infection towards long-term molecular latency as well as transition of latent infection to reactivation. $L$ and $R$ represent the LANA and Rta promoters, respectively. Active promoters are designated by black arrows, and inactive promoters are indicated by white arrows. Cylinders indicate chromatin marks (histones) of either repressive (orange) or active (green) nature. PRC, polycomb repressive complex; EZH2, enhancer of zeste 2 polycomb repressive complex 2 subunit; DNMT, DNA methyltransferase; TLK2, tousled-like kinase 2; JMJD, Jumonji domain-containing protein; UTX, ubiquitously transcribed tetratricopeptide repeat, X chromosome (lysine-specific demethylase).

The KSHV Rta protein (also known as ORF50) is necessary and sufficient to initiate KSHV reactivation $(105,106)$. Rta is a potent transcriptional activator that can bind DNA directly or through RBP-Jא (107). Rta reverses and overrides chromatinsilencing modifications, and deletion of Rta renders MHV-68 unable to reactivate from latency. In subsequent steps, other viral proteins such as K-bZIP augment the action of Rta to ensure robust and complete viral replication and virion formation. If Rta is the master regulator of reactivation from latency, what regulates Rta expression and Rta function? Here the experimental evidence is murkier. KSHV LANA and viral miRNAs counteract Rta and rapidly drive the virus into latency upon infection of primary endothelial cells, whereas in other environments Rta prevails (108-110). It is also worth mentioning that viral reactivation can occur in an Rta-independent fashion (111).

More research is needed to identify physiological triggers of KSHV reactivation as potential targets of disease prevention. These are likely to depend on conserved as well as cell- and microenvironment-specific signaling pathways (112). KSHV reactivation can be induced by IFN- $\gamma$, but not IFN- $\alpha$. KSHV reactivation is induced by TLR7/8 signaling, and reactivation is enhanced by deletion of RIG-I and MAVS $(38,39)$. In artificially infected Burkitt lymphoma B cells (BJAB cells), B cell receptor crosslinking can reactivate KSHV (113), though PELs are BCR negative. Different sets of events may trigger KSHV reactivation in the oral cavity versus endothelial cells.

\section{Update on LANA function and structure}

LANA binds the viral terminal repeats, specifically two sequenceconserved, high-affinity binding sites (LBS1 and LBS2) and a more divergent third, low-affinity site (114-116). LANA can be thought of as a dumbbell-like structure in which a stalk of internal repeats separates the two globular terminal regions. The stalk length is variable and nonessential for LANA's nuclear functions, as a direct N-to-C terminal domain fusion retains the latencysupporting functions. Whereas the C-terminal end of LANA binds KSHV DNA directly, the N-terminus (and perhaps regions in the C-terminus as well) contact cellular chromosome-associated proteins, such as histones H2A and H2B and others (104). The crystal structures of the KSHV LANA and MHV-68 LANA DNA-binding domains were solved $(102,103,116,117)$. This work identifies the DNA contact residues and reveals a folding pattern analogous to EBV EBNA1 and HPV E2.

LANA-episome complexes adopt higher-order structures in the nucleus of infected cells and appear as a characteristic punctate pattern by immunofluorescence $(114,118,119)$. Initially considered a somewhat underwhelming feature, these "LANA dots" have emerged as the diagnostic gold standard to identify KSHV-infected cells and to make the diagnosis of KS and PEL $(114,118,119)$. The number of LANA dots correlates with the number of KSHV plasmids in an infected cell. During mitosis, LANA, and by inference KSHV plasmids, decorates condensed chromosomes, thereby facilitating proper and equal partitioning of the latent viral genomes into daughter cells. Loss-of-function LANA mutants in the context of the viral genome remain competent for lytic replication, but fail to establish and maintain latency in KSHV and the related MHV68. Ablation of LANA in PEL is incompatible with growth. Thus, LANA can be considered essential for KSHV-associated lymphomagenesis. However, interpreting genetic experiments for LANA is rather complex, since tethering the KSHV plasmids to the host genome is not the only function of LANA. LANA also binds a large number of cellular proteins to modulate their functions, including p53 and many other proteins with specialized functions $(120,121)$. Most recently, cytoplasmic variants of LANA have been described (122), and whole-genome screens have highlighted the importance of LANA during KSHV primary infection $(45,47)$. 


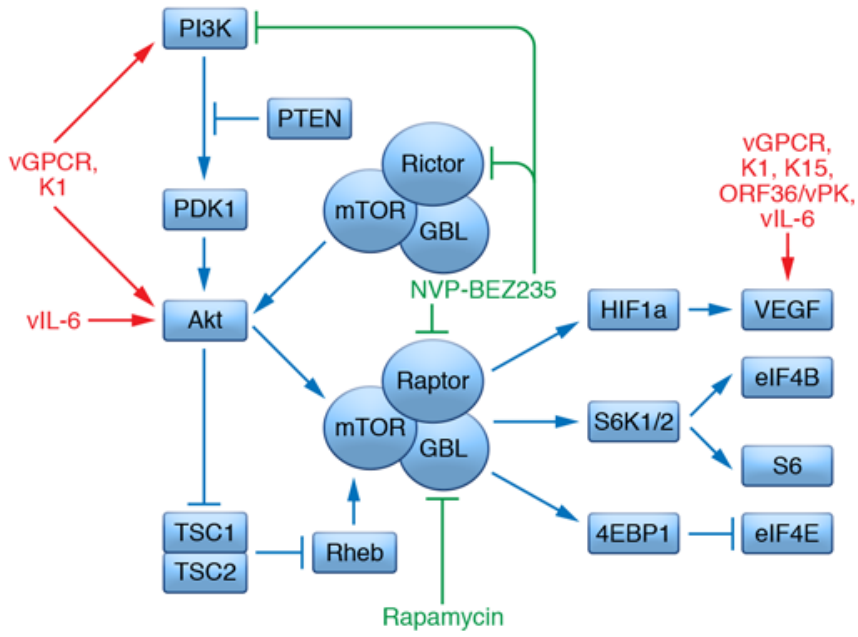

Figure 3. KSHV vGPCR, K1, K15, and vIL-6 proteins impact the PI3K/AKT/ mTOR signaling pathway. Multiple KSHV viral proteins activate the PI3K/ AKT/mTOR signaling pathway at different nodes in this pathway. Activation of this pathway ultimately results in cell survival and cell proliferation.

\section{Viral miRNAs support viral infection and latent persistence}

A recently emerged common feature among all herpesviruses is the utilization of virally encoded miRNAs as a means to modulate the host cell during latency and primary infection. In Marek's disease virus, a B cell-tropic alpha herpesvirus of chickens, viral miRNAs are the primary driver of oncogenesis. Recently, KSHV mir-K12-10a was identified as the molecular driver behind the in vitro transforming phenotype of KSHV Kaposin, since it is embedded within the open reading frame of this protein (123). The role of the KSHV miRNAs is often more subtle, but it is important to bear in mind that virally encoded miRNAs account for $50 \%$ or more of all miRNAs in a KSHV-infected B cell. KSHV encodes 12 pre-miRNA loci, which can give rise to 24 mature miRNAs and many more if alternative processing is considered (124-126). Many of the viral miRNAs are also secreted into pleural fluid and circulate in the blood of KS patients (127). Thus, they serve as biomarkers of latent infection.

In general, viral miRNAs target specific cellular mRNAs, leading to their degradation (via an siRNA-like mechanism) and inhibition of mRNA-directed translation. miRNAs are developmentally regulated and fine-tune lineage differentiation and cellular signaling. The targets of the KSHV miRNAs have been established through a series of comprehensive biochemical studies (128-131). Thus far, miRNA studies have been constrained by sensitivity limits for detection of individual miRNAs and for the discovery of miRNA-target interactions. Targets with functions that seem to befit the biology of B cell development, endothelial cell differentiation, and KSHV (such as BACH1, $\mathrm{xCT}, \mathrm{MAF}$, and others) have been individually validated (132-134). These are by no means the only targets, and it is anticipated that additional targets will be identified in the future.

Cellular miRNA-155 is central to B cell lineage development in the germinal center. Downregulation of miRNA-155 is associated with terminal differentiation of plasma cells and loss of proliferative potential. Conversely, ectopic expression of miRNA-155 is associated with hyperproliferation and lymphoma. $\mathrm{EBV}$ relies on endogenous miRNA-155 to drive lymphoblastoid cell line immortalization $(129,135)$. Similarly, KSHV encodes an ortholog of miRNA-155 named miRNA-K12-11 that contains 100\% seed sequence identity $(136,137)$. This KSHV ortholog complements the proliferative deficits observed in miR-155-deficient mice and drives lymphoma in a CD34 reconstitution model $(138,139)$. While miR155 is the first and best-studied viral ortholog of a cellular miRNA, it is not the only one. As our appreciation for the complexities of host cell miRNA function and regulation grows, we can expect to gain new insights into the biology of KSHV miRNAs as well.

\section{Genomic explorations of KS and PEL}

Only a small fraction of KSHV-infected children develop KS, just as only a small fraction of EBV-infected children develop Burkitt lymphoma. In the context of solid organ transplantation, only a fraction of KSHV-seropositive transplant recipients develop KS, similar to the small fraction of EBV-seropositive transplant recipients that develop posttransplant lymphoproliferative disease, a condition associated with EBV infection of B cells after therapeutic immunosuppression. Currently, there is no screening of organ donors for KSHV positivity, although screening for KSHV in donors is warranted. KS that develops in transplant patients is usually a late complication, developing several months after the onset of immune suppression therapy. By contrast, the onset of herpes simplex and CMV reactivation disease is more immediate, often necessitating acyclovir prophylaxis for the first 6 months after transplantation. The delayed emergence of KS vis-à-vis clinical diseases associated with these other herpesviruses suggests that in addition to KSHV and in addition to immune deficiency, genomic alterations may contribute to KSHV-associated neoplasia.

Family linkage studies in classic KS support the notion of susceptibility loci for KS (140-142). Whereas $\mathrm{t}(8 ; 14)$ and related translocations targeting MYC are the defining genomic event in EBV-related Burkitt's lymphoma, MYC translocations are not present in PEL. Rather, the KSHV viral protein LANA drives MYC overexpression $(143,144)$. Comparative genome hybridization uncovered fragile histidine triad (FHIT) deletion as overrepresented in PELs, and targeted sequencing studies identified a polymorphism in IL-1 receptor-associated kinase 1 (IRAK1) as significantly overrepresented in PELs $(145,146)$. Moreover, IRAK1 signaling is required for PEL growth. This observation parallels Waldenstrom macroglobulinemia and a fraction of diffuse large B cell lymphomas, where gain-of-function mutations in MyD88, the upstream partner of IRAK1, are present $(147,148)$. It is important, however, to recognize that the rarity of PEL and classic KS incidence hinders genomic explorations, which limits the statistical significance of any association.

PTEN, p53, and Rb are not deleted in PEL or KS; rather, they are inactivated posttranslationally, e.g., by direct binding to LANA, or via expression of the CDK1-resistant viral cyclin homo$\log$ vCYC (149). This may explain why KS is initially responsive to DNA-damaging chemotherapy. Susceptibility to etoposide correlates with p53 mutation status in PEL, and p53 activation by nutlin-3 leads to apoptosis $(120,121)$. In KS and PEL, the human genome is dynamic and the host mutational landscape is shaped by selection during clonal evolution of the tumor just as it is for 
non-infection-associated cancers. The presence of KSHV modifies a particular pattern of mutations, but these mutations affect the same progrowth and antiapoptosis pathways as in other cancers. However, the interpretation of signature mutations becomes complicated in light of their role in infection-associated cancers, such as PEL or KS. Whether a particular event has been selected for as a driver of tumorigenesis after viral infection or if it represents a susceptibility allele for the primary infection event (or asymptomatic, systemic persistence) is not always apparent.

Only recently have whole KSHV genome sequences become available from patients and primary biopsies $(150,151)$. These sequences augment extensive studies that trace the origin and evolution of KSHV based on single-gene analyses (152). KSHV sequences show overall structural concordance and limited variation, as would be expected since viral replication is the result of error-correcting, DNA-dependent DNA polymerases (the cellular DNA polymerase during latency and a viral KSHV-encoded DNA polymerase during lytic replication). During B cell latency, multiple copies of the KSHV plasmid are maintained, replicated by the host DNA polymerase, and propagated to daughter cells during host cell division events. As yet, there is no evidence for integration of the KSHV genome. Nevertheless, defective variants have been described and are expected to arise in the context of clonal expansion of PEL or advanced KS. KSHV noncoding regions such as the miRNA locus show more variation, and differences in miRNA sequences correlate with processing and function (153155). The number of terminal repeats in the KSHV genome is highly variable and can be used for strain typing $(156,157)$. Likewise, membrane proteins that are subject to immune recognition, such as $\mathrm{K} 1$ and $\mathrm{K} 15$, contain hypervariable regions in the extracellular domains (158-160).

\section{Targeted treatment approaches to KSHV- associated cancers}

KS is a disease of endothelial cells, and details of its pathobiology have been extensively reviewed. KS is among the most angiogenic cancers known to arise in humans. If we can decipher which factors drive KS and which treatments interrupt KS angiogenesis, then we will have potent leads for other cancers that depend on angiogenesis. VEGF, stem cell factor (SCF, also known as KIT ligand), and platelet-derived growth factor (PDGF) are the best-characterized paracrine drivers of KS angiogenesis $(161,162)$, and these are the target of a number of therapeutic approaches for KS. VEGFneutralizing antibodies (bevacizumab) and receptor tyrosine kinase (RTK) inhibitors, such as imatinib, have efficacy in KS (163, 164), although their therapeutic impact as single agents is limited because of redundancy in the paracrine network. Clinical studies have also started investigating the role of thalidomide, lenalidomide (NCT01057121), and pomalidomide (NCT02659930) in KS. These structurally related compounds are approved for the treatment of multiple myeloma and have antiinflammatory and antiangiogenic activities, though the exact molecular mechanisms underlying their effects have not necessarily been established. KSHV activates the PI3K/Akt/mTOR signaling pathway at different nodes via the viral proteins vGPCR, K1, ORF36/vPK, vIL-6, and $\mathrm{K} 15$, as well as through virus-mediated upregulation of cellular growth factors, e.g., VEGF and PDGF (165-172) (Figure 3).
PI3K activates the cell survival kinase AKT, which subsequently activates mTOR. Rapamycin (also known as sirolimus) has clinical activity against KS $(173,174)$. Switching from cyclosporine A to rapamycin as the primary immunosuppressant has become the first line of therapy for transplant KS. The clinical phenotype can be recapitulated in preclinical models of KS and PEL (175-179). In KS, targeting mTOR was associated with a decrease in VEGF production. In PEL, rapamycin reduced IL- 6 and IL-10 secretion, and inactivating PI3K and mTOR together had more potent antitumor activity than inhibiting mTOR alone (180). The latter findings provide a guide path for the development of next-generation PI3K/ AKT/mTOR targeting strategies against KS.

Therapies that target immunomodulatory mechanisms also hold promise for KS and KSHV-associated diseases. Siltuximab, a humanized anti-IL-6 antibody, has been FDA approved for classic Castleman's disease (181), and is likely to also show efficacy against MCD. A pilot clinical trial of tocilizumab, a humanized antibody against the IL-6 receptor, is open for MCD (NCT01441063). Blocking IL-6 stymies PEL growth in preclinical models (182). Hsp90 inhibitors exhibited nanomolar EC50 against PEL and KS in three independent studies (183-185). PELs are also extremely sensitive to NF- $\kappa$ B pathway inhibitors such as bortezomib $(186,187)$, and a clinical trial with adjuvant bortezomib is ongoing. Other targets with encouraging preclinical results are NOTCH (188-192), and the KSHV receptor, ephrin receptor A2 (EphA2) (193-195).

While some individual AIDS-KS lesions respond to combination antiretroviral therapy (cART) and the ensuing immune reconstitution, others do not. In the US, one third of KS cases now develop in HIV patients with no detectable HIV viral load and near-normal CD4 counts $(196,197)$. This type of KS no longer signifies terminal AIDS. In sub-Saharan Africa, where KS remains the most common disease among HIV patients and the most common AIDS-presenting symptom, initiating cART can lead to KS exacerbation in KS-IRIS $(9,10)$. At least two large clinical trials are currently underway to determine if it is better to give cART and chemotherapy sequentially or together, and which chemotherapy is best suited for which stage of KS $(198,199)$. Liposomal doxorubicin, daunorubicin, other anthracycline formulations, and taxol constitute the mainstay of KS treatment.

\section{The need for a better understanding of KSHV remains}

$\mathrm{KS}$ is the most common cancer in males in many African countries and remains the most common cancer in HIV-positive persons in countries where cART coverage is near universal. As with all diseases, a detailed molecular understanding of the primary etiologic agent, i.e., KSHV, forms the basis for the development of targeted therapeutics. If it is possible to cure latent HIV virus, it should also be possible to cure latent KSHV. LANA represents the most direct target for such an approach, although as described above, KSHV latency and KSHV persistence involve many viral proteins as well as viral miRNAs. Any of these proteins could become a clinically tractable target. A viral cure is limited without preventing subsequent reinfection. Pre-exposure prophylaxis may be possible for transplant patients or other at-risk populations, though the side effects of current antivirals (e.g., ganciclovir) are rather severe. A preventative vaccine would provide the best approach. 


\section{Is it time for a KSHV vaccine?}

We would argue that KSHV vaccine development is needed, and that both preventative and therapeutic KSHV vaccines would be of benefit. KSHV transmission among infants is similar to that of all other herpesviruses; by puberty, greater than $80 \%$ of children seroconvert in KSHV endemic regions. By contrast, transmission among adults in many parts of the world (excluding Africa and the Mediterranean) is so poor that repeated contact or immunodeficiency, as in high-risk populations, is needed to sustain the virus at a greater than $5 \%$ population-wide prevalence. This suggests that only a fraction of exposures leads to establishment of latency and eventual disease. Systemically circulating and salivary levels of KSHV in asymptomatic persons are orders of magnitude lower than those of EBV, herpes simplex virus, or human CMV $(13,14,200)$. Evidence of KSHV superinfection in immune-competent persons is limited. A little priming of the immune system by a vaccine prior to establishment of latency may be all that is needed to eradicate KSHV and KS-associated diseases from the human population.

\section{Acknowledgments}

We apologize for not citing many publications due to limits on reference numbers per journal policy. We thank members of the Dittmer and Damania labs and all our colleagues for stimulating discussions. Our work is supported by Public Health Service grants CA019014, CA163217, DE018304, DE023946, DE018281, and CA096500 from the National Cancer Institute (NCI) and the Institute of Dental and Craniofacial Research (NIDCR). DPD is an investigator of the AIDS malignancies clinical trial consortium (AMC). BD is a Leukemia and Lymphoma Society Scholar, and a Burroughs Wellcome Fund Investigator in Infectious Disease.

Address correspondence to: Blossom Damania, 450 West Drive, CB\# 7295, Rm 32-026, Lineberger Cancer Center, University of North Carolina at Chapel Hill, Chapel Hill, North Carolina 27599, USA. Phone: 919.843.6011; E-mail: damania@ med.unc.edu.
1. Silverberg MJ, et al. Cumulative incidence of cancer among persons with HIV in North America: a cohort study. Ann Intern Med. 2015;163(7):507-518.

2. Robbins HA, Pfeiffer RM, Shiels MS, Li J, Hall HI, Engels EA. Excess cancers among HIV-infected people in the United States. J Natl Cancer Inst. 2015;107(4): dju503.

3. Jemal A, et al. Cancer burden in Africa and opportunities for prevention. Cancer. 2012;118(18):4372-4384.

4. Moore PS, Chang Y. Detection of herpesvirus-like DNA sequences in Kaposi's sarcoma in patients with and without HIV infection. N Engl JMed. 1995;332(18):1181-1185.

5. Chang Y, et al. Identification of herpesvirus-like DNA sequences in AIDS-associated Kaposi's sarcoma. Science. 1994;266(5192):1865-1869.

6. Soulier J, et al. Kaposi's sarcoma-associated herpesvirus-like DNA sequences in multicentric Castleman's disease. Blood. 1995;86(4):1276-1280.

7. Nador RG, et al. Primary effusion lymphoma: a distinct clinicopathologic entity associated with the Kaposi's sarcoma-associated herpes virus. Blood. 1996;88(2):645-656.

8. Cesarman E, Chang Y, Moore PS, Said JW, Knowles DM. Kaposi's sarcoma-associated herpesvirus-like DNA sequences in AIDS-related body-cavity-based lymphomas. N Engl J Med. 1995;332(18):1186-1191.

9. Bower M, et al. Immune reconstitution inflammatory syndrome associated with Kaposi's sarcoma. J Clin Oncol. 2005;23(22):5224-5228.

10. Connick E, Kane MA, White IE, Ryder J, Campbell TB. Immune reconstitution inflammatory syndrome associated with Kaposi sarcoma during potent antiretroviral therapy. Clin Infect Dis. 2004;39(12):1852-1855

11. Polizzotto MN, et al. Clinical features and outcomes of patients with symptomatic Kaposi sarcoma herpesvirus (KSHV)-associated inflammation: prospective characterization of KSHV inflammatory cytokine syndrome (KICS). Clin Infect Dis. 2016;62(6):730-738.

12. Mayama S, et al. Prevalence and transmission of Kaposi's sarcoma-associated herpesvirus (human herpesvirus 8) in Ugandan children and adolescents. Int J Cancer. 1998;77(6):817-820.

13. Koelle DM, Huang ML, Chandran B, Vieira J, Piepkorn M, Corey L. Frequent detection of Kaposi's sarcoma-associated herpesvirus (human herpesvirus 8) DNA in saliva of human immunodeficiency virus-infected men: clinical and immunologic correlates. J Infect Dis. 1997;176(1):94-102.

14. Gantt S, et al. Reduced human herpesvirus-8 oropharyngeal shedding associated with protease inhibitor-based antiretroviral therapy. JClin Virol. 2014;60(2):127-132.

15. Wang L, Damania B. Kaposi's sarcoma-associated herpesvirus confers a survival advantage to endothelial cells. Cancer Res. 2008;68(12):4640-4648.

16. Mutlu $\mathrm{AD}$, et al. In vivo-restricted and reversible malignancy induced by human herpesvirus- 8 KSHV: a cell and animal model of virally induced Kaposi's sarcoma. Cancer Cell. 2007;11(3):245-258.

17. Jones T, et al. Direct and efficient cellular transformation of primary rat mesenchymal precursor cells by KSHV. JClin Invest. 2012;122(3):1076-1081.

18. An FQ, et al. Long-term-infected telomeraseimmortalized endothelial cells: a model for Kaposi's sarcoma-associated herpesvirus latency in vitro and in vivo. J Virol. 2006;80(10):4833-4846.

19. Lee MS, et al. Human mesenchymal stem cells of diverse origins support persistent infection with Kaposi's sarcoma-associated herpesvirus and manifest distinct angiogenic, invasive, and transforming phenotypes. MBio. 2016;7(1):e02109-e02115.

20. Dittmer D, et al. Experimental transmission of Kaposi's sarcoma-associated herpesvirus (KSHV/ HHV-8) to SCID-hu Thy/Liv mice. J Exp Med. 1999;190(12):1857-1868.

21. Sin SH, Dittmer DP. Viral latency locus augments $\mathrm{B}$-cell response in vivo to induce chronic marginal zone enlargement, plasma cell hyperplasia, and lymphoma. Blood. 2013;121(15):2952-2963.

22. Wang LX, et al. Humanized-BLT mouse model of Kaposi's sarcoma-associated herpesvirus infection. Proc Natl Acad Sci U S A. 2014;111(8):3146-3151.
23. Decker LL, et al. The Kaposi sarcoma-associated herpesvirus (KSHV) is present as an intact latent genome in KS tissue but replicates in the peripheral blood mononuclear cells of KS patients. J Exp Med.1996;184(1):283-288.

24. Akula SM, Wang FZ, Vieira J, Chandran B. Human herpesvirus 8 interaction with target cells involves heparan sulfate. Virology. 2001;282(2):245-255

25. Akula SM, Pramod NP, Wang FZ, Chandran B. Integrin alpha3beta1 (CD 49c/29) is a cellular receptor for Kaposi's sarcoma-associated herpesvirus (KSHV/HHV-8) entry into the target cells. Cell. 2002;108(3):407-419.

26. Wang FZ, Akula SM, Sharma-Walia N, Zeng L, Chandran B. Human herpesvirus 8 envelope glycoprotein B mediates cell adhesion via its RGD sequence. J Virol. 2003;77(5):3131-3147.

27. Rappocciolo G, et al. Human herpesvirus 8 infects and replicates in primary cultures of activated B lymphocytes through DC-SIGN.J Virol. 2008;82(10):4793-4806.

28. Rappocciolo G, et al. DC-SIGN is a receptor for human herpesvirus 8 on dendritic cells and macrophages. JImmunol. 2006;176(3):1741-1749.

29. Kaleeba JA, Berger EA. Kaposi's sarcoma-associated herpesvirus fusion-entry receptor: cystine transporter xCT. Science. 2006;311(5769):1921-1924.

30. Akula SM, Naranatt PP, Walia NS, Wang FZ, Fegley B, Chandran B. Kaposi's sarcoma-associated herpesvirus (human herpesvirus 8) infection of human fibroblast cells occurs through endocytosis. J Virol. 2003;77(14):7978-7990.

31. Chandran B. Early events in Kaposi's sarcomaassociated herpesvirus infection of target cells. J Virol. 2010;84(5):2188-2199.

32. Valiya Veettil M, Sadagopan S, Kerur N, Chakraborty S, Chandran B. Interaction of c-Cbl with myosin IIA regulates Bleb associated macropinocytosis of Kaposi's sarcoma-associated herpesvirus. PLoS Pathog. 2010;6(12):e1001238.

33. Ma Z, Damania B. The cGAS-STING defense pathway and its counteraction by viruses. Cell Host Microbe. 2016;19(2):150-158. 
34. Chen IY, Ichinohe T. Response of host inflammasomes to viral infection. Trends Microbiol. 2015;23(1):55-63.

35. West J, Damania B. Upregulation of the TLR3 pathway by Kaposi's sarcoma-associated herpesvirus during primary infection. J Virol. 2008;82(11):5440-5449.

36. West JA, Gregory SM, Sivaraman V, Su L, Damania B. Activation of plasmacytoid dendritic cells by Kaposi's sarcoma-associated herpesvirus. JVirol. 2011;85(2):895-904.

37. Lagos D, et al. Toll-like receptor 4 mediates innate immunity to Kaposi sarcoma herpesvirus. Cell Host Microbe. 2008;4(5):470-483.

38. Gregory SM, West JA, Dillon PJ, Hilscher C, Dittmer DP, Damania B. Toll-like receptor signaling controls reactivation of KSHV from latency. Proc Natl Acad Sci U S A. 2009;106(28):11725-11730.

39. West JA, et al. An important role for mitochondrial antiviral signaling protein in the Kaposi's sarcoma-associated herpesvirus life cycle. JVirol. 2014;88(10):5778-5787.

40. Inn KS, et al. Inhibition of RIG-I-mediated signaling by Kaposi's sarcoma-associated herpesvirus-encoded deubiquitinase ORF64. J Virol. 2011;85(20):10899-10904.

41. Jacobs SR, Damania B. NLRs, inflammasomes, and viral infection. J Leukoc Biol. 2012;92(3):469-477.

42. Gregory SM, et al. Discovery of a viral NLR homolog that inhibits the inflammasome. Science. 2011;331(6015):330-334

43. Kerur N, et al. IFI16 acts as a nuclear pathogen sensor to induce the inflammasome in response to Kaposi Sarcoma-associated herpesvirus infection. Cell Host Microbe. 2011;9(5):363-375.

44. Singh VV, et al. Kaposi's sarcoma-associated herpesvirus latency in endothelial and B cells activates gamma interferon-inducible protein 16-mediated inflammasomes. J Virol. 2013;87(8):4417-4431.

45. Ma Z, et al. Modulation of the cGAS-STING DNA sensing pathway by gammaherpesviruses. Proc Natl Acad Sci U S A. 2015;112(31):E4306-E4315.

46. Wu JJ, et al. Inhibition of cGAS DNA sensing by a herpesvirus virion protein. Cell Host Microbe. 2015;18(3):333-344

47. Zhang G, et al. Cytoplasmic isoforms of Kaposi sarcoma herpesvirus LANA recruit and antagonize the innate immune DNA sensor cGAS. Proc Natl Acad Sci U S A. 2016;113(8):E1034-E1043.

48. Jacobs SR, Damania B. The viral interferon regulatory factors of KSHV: immunosuppressors or oncogenes? Front Immunol. 2011;2:19.

49. Jacobs SR, et al. The viral interferon regulatory factors of kaposi's sarcoma-associated herpesvirus differ in their inhibition of interferon activation mediated by toll-like receptor 3.J Virol. 2013;87(2):798-806

50. Hwang KY, Choi YB. Modulation of mitochondrial antiviral signaling by human herpesvirus 8 interferon regulatory factor 1 . J Virol. 2016;90(1):506-520.

51. Jacobs SR, Stopford CM, West JA, Bennett CL, Giffin L, Damania B. Kaposi's sarcomaassociated herpesvirus viral interferon regulatory factor 1 interacts with a member of the interferon-stimulated gene 15 pathway. J Virol. 2015;89(22):11572-11583.
52. Spiller OB, Blackbourn DJ, Mark L, Proctor DG, Blom AM. Functional activity of the complement regulator encoded by Kaposi's sarcoma-associated herpesvirus. J Biol Chem. 2003;278(11):9283-9289.

53. Spiller OB, et al. Complement regulation by Kaposi's sarcoma-associated herpesvirus ORF4 protein. J Virol. 2003;77(1):592-599.

54. Lee MS, Jones T, Song DY, Jang JH, Jung JU, Gao SJ. Exploitation of the complement system by oncogenic Kaposi's sarcoma-associated herpesvirus for cell survival and persistent infection. PLoS Pathog. 2014;10(9):e1004412.

55. Liang Q, Fu B, Wu F, Li X, Yuan Y, Zhu F. ORF45 of Kaposi's sarcoma-associated herpesvirus inhibits phosphorylation of interferon regulatory factor 7 by IKK $\varepsilon$ and TBK1 as an alternative substrate. J Virol. 2012;86(18):10162-10172.

56. Zhu FX, Sathish N, Yuan Y. Antagonism of host antiviral responses by Kaposi's sarcoma-associated herpesvirus tegument protein ORF45. PLOS One. 2010;5(5):e10573.

57. González CM, Wang L, Damania B. Kaposi's sarcoma-associated herpesvirus encodes a viral deubiquitinase. J Virol. 2009;83(19):10224-10233.

58. Zhao Q, et al. Kaposi's sarcoma-associated herpesvirus-encoded replication and transcription activator impairs innate immunity via ubiquitinmediated degradation of myeloid differentiation factor 88. J Virol. 2015;89(1):415-427.

59. Ahmad H, et al. Kaposi sarcoma-associated herpesvirus degrades cellular Toll-interleukin-1 receptor domain-containing adaptorinducing beta-interferon (TRIF). J Biol Chem. 2011;286(10):7865-7872.

60. Yu Y, Hayward GS. The ubiquitin E3 ligase RAUL negatively regulates type i interferon through ubiquitination of the transcription factors IRF7 and IRF3. Immunity. 2010;33(6):863-877.

61. Lingel A, et al. Kaposi's sarcoma-associated herpesvirus reduces cellular myeloid differentiation primary-response gene 88 (MyD88) expression via modulation of its RNA. J Virol. 2016;90(1):180-188.

62. Cloutier N, Flamand L. Kaposi sarcoma-associated herpesvirus latency-associated nuclear antigen inhibits interferon (IFN) beta expression by competing with IFN regulatory factor-3 for binding to IFNB promoter. J Biol Chem. 2010;285(10):7208-7221.

63. Lu F, et al. Identification of host-chromosome binding sites and candidate gene targets for Kaposi's sarcoma-associated herpesvirus LANA. JVirol. 2012;86(10):5752-5762.

64. Ribechini E, et al. Identification of $\mathrm{CD} 8^{+} \mathrm{T}$ cell epitopes within lytic antigens of human herpes virus 8. J Immunol. 2006;176(2):923-930.

65. Stebbing J, et al. Kaposi's sarcoma-associated herpesvirus cytotoxic $\mathrm{T}$ lymphocytes recognize and target Darwinian positively selected autologous K1 epitopes. J Virol. 2003;77(7):4306-4314.

66. Wang $\mathrm{QJ}$, et al. Identification of an $\mathrm{HLA} \mathrm{A}^{*} \mathrm{O2O1}-$ restricted CD8(+) T-cell epitope for the glycoprotein B homolog of human herpesvirus 8. Blood. 2002;99(9):3360-3366.

67. Bekerman E, Jeon D, Ardolino M, Coscoy L. A role for host activation-induced cytidine deaminase in innate immune defense against KSHV. PLoS Pathog. 2013;9(11):e1003748.
68. Coscoy L, Ganem D. Kaposi's sarcoma-associated herpesvirus encodes two proteins that block cell surface display of MHC class I chains by enhancing their endocytosis. Proc Natl Acad Sci US A. 2000;97(14):8051-8056.

69. Brulois K, et al. Kaposi's sarcoma-associated herpesvirus K3 and K5 ubiquitin E3 ligases have stage-specific immune evasion roles during lytic replication. J Virol. 2014;88(16):9335-9349.

70. Stevenson PG, Efstathiou S, Doherty PC, Lehner PJ. Inhibition of MHC class I-restricted antigen presentation by gamma 2-herpesviruses. Proc Natl Acad Sci U S A. 2000;97(15):8455-8460.

71. Ishido S, Wang C, Lee BS, Cohen GB, Jung JU. Downregulation of major histocompatibility complex class I molecules by Kaposi's sarcomaassociated herpesvirus $\mathrm{K} 3$ and $\mathrm{K} 5$ proteins. JVirol. 2000;74(11):5300-5309.

72. Lindow M, et al. The virus-encoded chemokine vMIP-II inhibits virus-induced Tc1-driven inflammation. J Virol. 2003;77(13):7393-7400.

73. Li Q, Means R, Lang S, Jung JU. Downregulation of gamma interferon receptor 1 by Kaposi's sarcoma-associated herpesvirus K3 and K5. J Virol. 2007;81(5):2117-2127.

74. Coscoy L, Ganem D. A viral protein that selectively downregulates ICAM-1 and B7-2 and modulates $\mathrm{T}$ cell costimulation. J Clin Invest. 2001;107(12):1599-1606

75. Ishido $S$, et al. Inhibition of natural killer cellmediated cytotoxicity by Kaposi's sarcomaassociated herpesvirus $\mathrm{K} 5$ protein. Immunity. 2000;13(3):365-374.

76. Thomas M, et al. Down-regulation of NKG2D and NKp80 ligands by Kaposi's sarcomaassociated herpesvirus $\mathrm{K} 5$ protects against NK cell cytotoxicity. Proc Natl Acad Sci U S A. 2008;105(5):1656-1661.

77. Rezaee SA, Gracie JA, McInnes IB, Blackbourn DJ. Inhibition of neutrophil function by the Kaposi's sarcoma-associated herpesvirus vOX2 protein. AIDS. 2005;19(16):1907-1910.

78. Foster-Cuevas M, Wright GJ, Puklavec MJ, Brown MH, Barclay AN. Human herpesvirus 8 K14 protein mimics $\mathrm{CD} 200$ in down-regulating macrophage activation through CD200 receptor. JVirol. 2004;78(14):7667-7676.

79. Misstear K, et al. Suppression of antigen-specific $\mathrm{T}$ cell responses by the Kaposi's sarcomaassociated herpesvirus viral OX2 protein and its cellular orthologue, CD200. J Virol. 2012;86(11):6246-6257.

80. Chatterjee M, Osborne J, Bestetti G, Chang Y, Moore PS. Viral IL-6-induced cell proliferation and immune evasion of interferon activity. Science. 2002;298(5597):1432-1435.

81. Kledal TN, et al. A broad-spectrum chemokine antagonist encoded by Kaposi's sarcoma-associated herpesvirus. Science. 1997;277(5332):1656-1659.

82. Yamin R, et al. The viral KSHV chemokine vMIP-II inhibits the migration of Naive and activated human NK cells by antagonizing two distinct chemokine receptors. PLoS Pathog. 2013;9(8):e1003568.

83. Sozzani S, et al. The viral chemokine macrophage inflammatory protein-II is a selective Th2 chemoattractant. Blood. 1998;92(11):4036-4039.

84. Stine JT, et al. KSHV-encoded CC chemokine 
vMIP-III is a CCR4 agonist, stimulates angiogenesis, and selectively chemoattracts TH2 cells. Blood. 2000;95(4):1151-1157.

85. Myoung J, Ganem D. Infection of primary human tonsillar lymphoid cells by KSHV reveals frequent but abortive infection of T cells. Virology. 2011;413(1):1-11.

86. Gregory SM, Wang L, West JA, Dittmer DP, Damania B. Latent Kaposi's sarcoma-associated herpesvirus infection of monocytes downregulates expression of adaptive immune response costimulatory receptors and proinflammatory cytokines. J Virol. 2012;86(7):3916-3923.

87. Wu W, et al. KSHV/HHV-8 infection of human hematopoietic progenitor $\left(\mathrm{CD} 34^{+}\right)$cells: persistence of infection during hematopoiesis in vitro and in vivo. Blood. 2006;108(1):141-51.

88. Parsons CH, et al. KSHV targets multiple leukocyte lineages during long-term productive infection in NOD/SCID mice. J Clin Invest. 2006;116(7):1963-1973.

89. Speck SH, Ganem D. Viral latency and its regulation: lessons from the gamma-herpesviruses. Cell Host Microbe. 2010;8(1):100-115.

90. Feldman ER, et al. Virus-encoded microRNAs facilitate gammaherpesvirus latency and pathogenesis in vivo. MBio. 2014;5(3):e00981-e00914.

91. Mboko WP, et al. Tumor suppressor interferonregulatory factor 1 counteracts the germinal center reaction driven by a cancer-associated gammaherpesvirus. J Virol. 2016;90(6):2818-2829.

92. Diebel KW, et al. Gammaherpesvirus small noncoding RNAs are bifunctional elements that regulate infection and contribute to virulence in vivo. MBio. 2015;6(1):e01670-e01614.

93. Bhatt S, et al. Efficacious proteasome/HDAC inhibitor combination therapy for primary effusion lymphoma. JClin Invest. 2013;123(6):2616-2628.

94. Lechowicz M, et al. Molecular and clinical assessment in the treatment of AIDS Kaposi sarcoma with valproic acid. Clin Infect Dis. 2009;49(12):1946-1949.

95. Shin HJ, DeCotiis J, Giron M, Palmeri D, Lukac DM. Histone deacetylase classes I and II regulate Kaposi's sarcoma-associated herpesvirus reactivation. J Virol. 2014;88(2):1281-1292.

96. Darst RP, Haecker I, Pardo CE, Renne R, Kladde MP. Epigenetic diversity of Kaposi's sarcomaassociated herpesvirus. Nucleic Acids Res. 2013;41(5):2993-3009.

97. Renne R, et al. Lytic growth of Kaposi's sarcomaassociated herpesvirus (human herpesvirus 8 ) in culture. Nat Med.1996;2(3):342-346.

98. Günther T, Grundhoff A. The epigenetic landscape of latent Kaposi sarcomaassociated herpesvirus genomes. PLoS Pathog. 2010;6(6):e1000935.

99. Toth Z, et al. Biphasic euchromatin-to-heterochromatin transition on the KSHV genome following de novo infection. PLoS Pathog. 2013;9(12):e1003813.

100.Kang H, Wiedmer A, Yuan Y, Robertson E, Lieberman PM. Coordination of KSHV latent and lytic gene control by CTCF-cohesin mediated chromosome conformation. PLoS Pathog. 2011;7(8):e1002140.

101. Hilton IB, Simon JM, Lieb JD, Davis IJ, Damania $\mathrm{B}$, Dittmer DP. The open chromatin landscape of
Kaposi's sarcoma-associated herpesvirus. J Virol. 2013;87(21):11831-11842.

102. Domsic JF, Chen HS, Lu F, Marmorstein

R, Lieberman PM. Molecular basis for

oligomeric-DNA binding and episome maintenance by KSHV LANA. PLoS Pathog. 2013;9(10):e1003672.

103. Hellert J, et al. A structural basis for BRD2/4mediated host chromatin interaction and oligomer assembly of Kaposi sarcoma-associated herpesvirus and murine gammaherpesvirus LANA proteins. PLoS Pathog. 2013;9(10):e1003640.

104.Barbera AJ, et al. The nucleosomal surface as a docking station for Kaposi's sarcoma herpesvirus LANA. Science. 2006;311(5762):856-861.

105. Lukac DM, Renne R, Kirshner JR, Ganem D. Reactivation of Kaposi's sarcoma-associated herpesvirus infection from latency by expression of the ORF 50 transactivator, a homolog of the EBV R protein. Virology. 1998;252(2):304-312.

106.Sun R, Lin SF, Gradoville L, Yuan Y, Zhu F, Miller $G$. A viral gene that activates lytic cycle expression of Kaposi's sarcoma-associated herpesvirus. Proc Natl Acad Sci U S A. 1998;95(18):10866-10871.

107. Liang Y, Chang J, Lynch SJ, Lukac DM, Ganem D. The lytic switch protein of KSHV activates gene expression via functional interaction with RBPJkappa (CSL), the target of the Notch signaling pathway. Genes Dev. 2002;16(15):1977-1989.

108. Bellare P, Ganem D. Regulation of KSHV lytic switch protein expression by a virus-encoded microRNA: an evolutionary adaptation that fine-tunes lytic reactivation. Cell Host Microbe. 2009;6(6):570-575.

109. Lan K, Kuppers DA, Verma SC, Robertson ES. Kaposi's sarcoma-associated herpesvirus-encoded latency-associated nuclear antigen inhibits lytic replication by targeting Rta: a potential mechanism for virus-mediated control of latency. JVirol. 2004;78(12):6585-6594.

110.Jha HC, Lu J, Verma SC, Banerjee S, Mehta D, Robertson ES. Kaposi's sarcoma-associated herpesvirus genome programming during the early stages of primary infection of peripheral blood mononuclear cells. MBio. 2014;5(6):e02261-14.

111. Prasad A, Lu M, Lukac DM, Zeichner SL. An alternative Kaposi's sarcoma-associated herpesvirus replication program triggered by host cell apoptosis. J Virol. 2012;86(8):4404-4419.

112. Yu F, et al. Systematic identification of cellular signals reactivating Kaposi sarcoma-associated herpesvirus. PLoS Pathog. 2007;3(3):e44.

113. Kati $S$, et al. Activation of the $B$ cell antigen receptor triggers reactivation of latent Kaposi's sarcoma-associated herpesvirus in B cells. J Virol. 2013;87(14):8004-8016.

114. Ballestas ME, Chatis PA, Kaye KM. Efficient persistence of extrachromosomal KSHV DNA mediated by latency-associated nuclear antigen. Science. 1999;284(5414):641-644.

115. Hu J, Renne R. Characterization of the minimal replicator of Kaposi's sarcoma-associated herpesvirus latent origin. J Virol. 2005;79(4):2637-2642.

116. Hellert J, et al. The 3D structure of Kaposi sarcoma herpesvirus LANA C-terminal domain bound to DNA. Proc Natl Acad Sci USA. 2015;112(21):6694-6699.

117. Correia B, et al. Crystal structure of the gamma-2 herpesvirus LANA DNA binding domain identifies charged surface residues which impact viral latency. PLoS Pathog. 2013;9(10):e1003673.

118. Kedes DH, Lagunoff M, Renne R, Ganem D. Identification of the gene encoding the major latency-associated nuclear antigen of the Kaposi's sarcoma-associated herpesvirus. J Clin Invest. 1997;100(10):2606-2610.

119. Kellam P, Boshoff C, Whitby D, Matthews S, Weiss RA, Talbot SJ. Identification of a major latent nuclear antigen, LNA-1, in the human herpesvirus 8 genome. J Hum Virol. 1997;1(1):19-29.

120. Sarek G, et al. Reactivation of the $\mathrm{p} 53$ pathway as a treatment modality for KSHV-induced lymphomas. J Clin Invest. 2007;117(4):1019-1028.

121. Petre CE, Sin SH, Dittmer DP. Functional p53 signaling in Kaposi's sarcoma-associated herpesvirus lymphomas: implications for therapy. J Virol. 2007;81(4):1912-1922.

122. Kwun HJ, Toptan T, Ramos da Silva S, Atkins JF, Moore PS, Chang Y. Human DNA tumor viruses generate alternative reading frame proteins through repeat sequence recoding. Proc Natl Acad Sci U S A. 2014;111(41):E4342-E4349.

123. Forte $\mathrm{E}$, et al. MicroRNA-mediated transformation by the Kaposi's sarcoma-associated herpesvirus Kaposin locus. J Virol. 2015;89(4):2333-2341.

124.Samols MA, Hu J, Skalsky RL, Renne R. Cloning and identification of a microRNA cluster within the latency-associated region of Kaposi's sarcoma-associated herpesvirus. J Virol. 2005;79(14):9301-9305.

125. Cai X, Lu S, Zhang Z, Gonzalez CM, Damania B, Cullen BR. Kaposi's sarcoma-associated herpesvirus expresses an array of viral microRNAs in latently infected cells. Proc Natl Acad Sci U S A. 2005;102(15):5570-5575.

126.Pfeffer S, et al. Identification of microRNAs of the herpesvirus family. Nat Methods. 2005;2(4):269-276.

127. Chugh PE, et al. Systemically circulating viral and tumor-derived microRNAs in KSHV-associated malignancies. PLoS Pathog. 2013;9(7):e1003484.

128. Haecker I, et al. Ago HITS-CLIP expands understanding of Kaposi's sarcoma-associated herpesvirus miRNA function in primary effusion lymphomas. PLoS Pathog. 2012;8(8):e1002884.

129. Gottwein E, et al. Viral microRNA targetome of KSHV-infected primary effusion lymphoma cell lines. Cell Host Microbe. 2011;10(5):515-526.

130.Ziegelbauer JM, Sullivan CS, Ganem D. Tandem array-based expression screens identify host mRNA targets of virus-encoded microRNAs. Nat Genet. 2009;41(1):130-134.

131. Ziegelbauer JM. Viral microRNA genomics and target validation. Curr Opin Virol. 2014;7:33-39.

132. Hansen A, et al. KSHV-encoded miRNAs target MAF to induce endothelial cell reprogramming. Genes Dev. 2010;24(2):195-205.

133. Qin Z, et al. Upregulation of $x C T$ by KSHVencoded microRNAs facilitates KSHV dissemination and persistence in an environment of oxidative stress. PLoS Pathog. 2010;6(1):e1000742.

134. Hu M, et al. A KSHV microRNA directly targets $G$ protein-coupled receptor kinase 2 to promote the migration and invasion of endothelial cells by inducing CXCR2 and activating AKT signaling. PLoS Pathog. 2015;11(9):e1005171. 
135. Linnstaedt SD, Gottwein E, Skalsky RL, Luftig MA, Cullen BR. Virally induced cellular microRNA miR-155 plays a key role in B-cell immortalization by Epstein-Barr virus. J Virol. 2010;84(22):11670-11678.

136. Gottwein $\mathrm{E}$, et al. A viral microRNA functions as an orthologue of cellular miR-155. Nature. 2007;450(7172):1096-1099.

137. Skalsky RL, et al. Kaposi's sarcoma-associated herpesvirus encodes an ortholog of miR-155. JVirol. 2007;81(23):12836-12845.

138. Boss IW, Nadeau PE, Abbott JR, Yang Y, Mergia A, Renne R. A Kaposi's sarcoma-associated herpesvirus-encoded ortholog of microRNA miR-155 induces human splenic B-cell expansion in NOD/LtSz-scid IL2Rynull mice. JVirol. 2011;85(19):9877-9886.

139. Sin SH, Kim YB, Dittmer DP. Latency locus complements MicroRNA 155 deficiency in vivo. JVirol. 2013;87(21):11908-11911.

140. Guttman-Yassky E, et al. Familial clustering of classic Kaposi sarcoma. J Infect Dis. 2004;189(11):2023-2026.

141. Byun M, et al. Inherited human OX40 deficiency underlying classic Kaposi sarcoma of childhood. JExp Med. 2013;210(9):1743-1759.

142.Byun $\mathrm{M}$, et al. Whole-exome sequencingbased discovery of STIM1 deficiency in a child with fatal classic Kaposi sarcoma. J Exp Med. 2010;207(11):2307-2312.

143. Bubman D, Guasparri I, Cesarman E. Deregulation of c-Myc in primary effusion lymphoma by Kaposi's sarcoma herpesvirus latency-associated nuclear antigen. Oncogene. 2007;26(34):4979-4986.

144.Liu J, Martin HJ, Liao G, Hayward SD. The Kaposi's sarcoma-associated herpesvirus LANA protein stabilizes and activates c-Myc. JVirol. 2007;81(19):10451-10459.

145. Yang D, Chen W, Xiong J, Sherrod CJ, Henry DH, Dittmer DP. Interleukin 1 receptorassociated kinase 1 (IRAK1) mutation is a common, essential driver for Kaposi sarcoma herpesvirus lymphoma. Proc Natl Acad Sci U S A. 2014;111(44):E4762-E4768.

146.Roy D, Sin SH, Damania B, Dittmer DP. Tumor suppressor genes FHIT and WWOX are deleted in primary effusion lymphoma (PEL) cell lines. Blood. 2011;118(7):e32-e39.

147. Treon SP, et al. MYD88 L265P somatic mutation in Waldenström's macroglobulinemia. $N$ Engl J Med. 2012;367(9):826-833.

148. Ngo VN, et al. Oncogenically active MYD88 mutations in human lymphoma. Nature. 2011;470(7332):115-119.

149.Swanton C, Mann DJ, Fleckenstein B, Neipel F, Peters G, Jones N. Herpes viral cyclin/Cdk6 complexes evade inhibition by CDK inhibitor proteins. Nature. 1997;390(6656):184-187.

150. Tamburro KM, et al. Vironome of Kaposi sarcoma associated herpesvirus-inflammatory cytokine syndrome in an AIDS patient reveals co-infection of human herpesvirus 8 and human herpesvirus 6A. Virology. 2012;433(1):220-225.

151. Olp LN, Jeanniard A, Marimo C, West JT, Wood C. Whole-genome sequencing of Kaposi's sarcoma-associated herpesvirus from Zambian Kaposi's sarcoma biopsy specimens reveals unique viral diversity. J Virol. 2015;89(24):12299-12308.
152. Hayward GS, Zong JC. Modern evolutionary history of the human KSHV genome. Curr Top Microbiol Immunol. 2007;312:1-42.

153. Han SJ, et al. Kaposi's sarcoma-associated herpesvirus microRNA single-nucleotide polymorphisms identified in clinical samples can affect microRNA processing, level of expression, and silencing activity. JVirol. 2013;87(22):12237-12248.

154. Gottwein E, Cai X, Cullen BR. A novel assay for viral microRNA function identifies a single nucleotide polymorphism that affects Drosha processing. JVirol. 2006;80(11):5321-5326.

155. Ray A, et al. Sequence analysis of Kaposi sarcoma-associated herpesvirus (KSHV) microRNAs in patients with multicentric Castleman disease and KSHV-associated inflammatory cytokine syndrome. J Infect Dis. 2012;205(11):1665-1676.

156. Lagunoff M, Ganem D. The structure and coding organization of the genomic termini of Kaposi's sarcoma-associated herpesvirus. Virology. 1997;236(1):147-154.

157. Boulanger E, Duprez R, Delabesse E, Gabarre J, Macintyre E, Gessain A. Mono/oligoclonal pattern of Kaposi Sarcoma-associated herpesvirus (KSHV/HHV-8) episomes in primary effusion lymphoma cells. Int J Cancer. 2005;115(4):511-518.

158. Lacoste V, et al. Molecular epidemiology of human herpesvirus 8 in Africa: both B and A5 $\mathrm{K} 1$ genotypes, as well as the $\mathrm{M}$ and $\mathrm{P}$ genotypes of K14.1/K15 loci, are frequent and widespread. Virology. 2000;278(1):60-74.

159. Mbulaiteye S, et al. Molecular evidence for mother-to-child transmission of Kaposi sarcoma-associated herpesvirus in Uganda and K1 gene evolution within the host. Infect Dis. 2006;193(9):1250-1257.

160. White T, et al. Genetic diversity of the Kaposi's sarcoma herpesvirus K1 protein in AIDS-KS in Zimbabwe. JClin Virol. 2008;42(2):165-171.

161. Moses AV, et al. Kaposi's sarcoma-associated herpesvirus-induced upregulation of the c-kit proto-oncogene, as identified by gene expression profiling, is essential for the transformation of endothelial cells. J Virol. 2002;76(16):8383-8399.

162.Cavallin LE, Goldschmidt-Clermont P, Mesri EA. Molecular and cellular mechanisms of KSHV oncogenesis of Kaposi's sarcoma associated with HIV/AIDS. PLoS Pathog. 2014;10(7):e1004154.

163. Koon HB, et al. Phase II trial of imatinib in AIDS-associated Kaposi's sarcoma: AIDS Malignancy Consortium Protocol 042. JClin Oncol. 2014;32(5):402-408.

164. Uldrick TS, et al. Phase II study of bevacizumab in patients with HIV-associated Kaposi's sarcoma receiving antiretroviral therapy. JClin Oncol. 2012;30(13):1476-1483.

165. Bais C, et al. G-protein-coupled receptor of Kaposi's sarcoma-associated herpesvirus is a viral oncogene and angiogenesis activator. Nature. 1998;391(6662):86-89.

166. Wang L, et al. The Kaposi's sarcoma-associated herpesvirus (KSHV/HHV-8) K1 protein induces expression of angiogenic and invasion factors. Cancer Res. 2004;64(8):2774-2781.

167. Wang L, Dittmer DP, Tomlinson CC, Fakhari FD, Damania B. Immortalization of primary endothelial cells by the K1 protein of Kaposi's sarcoma-associated herpesvirus. Cancer Res.
2006;66(7):3658-3666.

168. Lee H, et al. Deregulation of cell growth by the K1 gene of Kaposi's sarcoma-associated herpesvirus. Nat Med.1998;4(4):435-440.

169.Bala K, et al. Kaposi's sarcoma herpesvirus K15 protein contributes to virus-induced angiogenesis by recruiting PLC $\gamma 1$ and activating NFAT1dependent RCAN1 expression. PLoS Pathog. 2012;8(9):e1002927.

170.Zhang Z, Chen W, Sanders MK, Brulois KF, Dittmer DP, Damania B. The K1 protein of Kaposi's sarcoma-associated herpesvirus (KSHV) augments viral lytic replication [published online ahead of print June 15, 2016]. JVirol. doi:10.1128/ JVI.03102-15.

171. Bhatt AP, et al. A viral kinase mimics $S 6$ kinase to enhance cell proliferation. Proc Natl Acad Sci U S A. 2016;113(28):7876-7881.

172. Morris VA, Punjabi AS, Wells RC, Wittkopp CJ, Vart R, Lagunoff M. The KSHV viral IL-6 homolog is sufficient to induce blood to lymphatic endothelial cell differentiation. Virology. 2012;428(2):112-120.

173. Krown SE, et al. Rapamycin with antiretroviral therapy in AIDS-associated Kaposi sarcoma: an AIDS Malignancy Consortium study. J Acquir Immune Defic Syndr. 2012;59(5):447-454.

174. Stallone G, et al. Sirolimus for Kaposi's sarcoma in renal-transplant recipients. $N$ Engl JMed. 2005;352(13):1317-1323.

175. Roy D, et al. mTOR inhibitors block Kaposi sarcoma growth by inhibiting essential autocrine growth factors and tumor angiogenesis. Cancer Res. 2013;73(7):2235-2246.

176. Sodhi A, et al. The TSC2/mTOR pathway drives endothelial cell transformation induced by the Kaposi's sarcoma-associated herpesvirus $\mathrm{G}$ protein-coupled receptor. Cancer Cell. 2006;10(2):133-143.

177. Nichols LA, Adang LA, Kedes DH. Rapamycin blocks production of KSHV/HHV8: insights into the anti-tumor activity of an immunosuppressant drug. PLoS One. 2011;6(1):e14535.

178. Chang HH, Ganem D. A unique herpesviral transcriptional program in KSHV-infected lymphatic endothelial cells leads to mTORC1 activation and rapamycin sensitivity. Cell Host Microbe. 2013;13(4):429-440.

179. Sin SH, et al. Rapamycin is efficacious against primary effusion lymphoma (PEL) cell lines in vivo by inhibiting autocrine signaling. Blood. 2007;109(5):2165-2173.

180. Bhatt AP, Bhende PM, Sin SH, Roy D, Dittmer DP, Damania B. Dual inhibition of PI3K and mTOR inhibits autocrine and paracrine proliferative loops in PI3K/Akt/mTOR-addicted lymphomas. Blood. 2010;115(22):4455-4463.

181. van Rhee F, et al. Siltuximab for multicentric Castleman's disease: a randomised, doubleblind, placebo-controlled trial. Lancet Oncol. 2014;15(9):966-974.

182. Jones KD, Aoki Y, Chang Y, Moore PS, Yarchoan $\mathrm{R}$, Tosato G. Involvement of interleukin-10 (IL10) and viral IL-6 in the spontaneous growth of Kaposi's sarcoma herpesvirus-associated infected primary effusion lymphoma cells. Blood. 1999;94(8):2871-2879.

183. Chen W, Sin SH, Wen KW, Damania B, Dittmer 
DP. Hsp90 inhibitors are efficacious against Kaposi Sarcoma by enhancing the degradation of the essential viral gene LANA, of the viral coreceptor EphA2 as well as other client proteins. PLoS Pathog. 2012;8(11):e1003048.

184. Nayar U, et al. Targeting the Hsp90-associated viral oncoproteome in gammaherpesvirus-associated malignancies. Blood. 2013;122(16):2837-2847.

185. Gopalakrishnan R, Matta H, Chaudhary PM. A purine scaffold HSP9O inhibitor BIIBO21 has selective activity against KSHV-associated primary effusion lymphoma and blocks vFLIP K13-induced NF-kB. Clin Cancer Res. 2013;19(18):5016-5026.

186. Keller SA, Schattner EJ, Cesarman E. Inhibition of NF-kappaB induces apoptosis of KSHVinfected primary effusion lymphoma cells. Blood. 2000;96(7):2537-2542.

187. Sarosiek KA, et al. Efficacy of bortezomib in a direct xenograft model of primary effusion lymphoma. Proc Natl Acad Sci U S A. 2010;107(29):13069-13074.

188. Liu R, et al. KSHV-induced notch components render endothelial and mural cell characteristics and cell survival. Blood. 2010;115(4):887-895.
189.Wang X, et al. Latency-associated nuclear antigen of Kaposi sarcoma-associated herpesvirus promotes angiogenesis through targeting notch signaling effector Hey1. Cancer Res. 2014;74(7):2026-2037.

190.Gasperini P, et al. Kaposi sarcoma herpesvirus promotes endothelial-to-mesenchymal transition through Notch-dependent signaling. Cancer Res. 2012;72(5):1157-1169.

191. Cheng F, et al. KSHV-initiated notch activation leads to membrane-type-1 matrix metalloproteinase-dependent lymphatic endothelial-tomesenchymal transition. Cell Host Microbe. 2011;10(6):577-590.

192.Curry CL, Reed LL, Golde TE, Miele L, Nickoloff BJ, Foreman KE. Gamma secretase inhibitor blocks Notch activation and induces apoptosis in Kaposi's sarcoma tumor cells. Oncogene. 2005;24(42):6333-6344.

193. Scehnet JS, et al. The role of Ephs, Ephrins, and growth factors in Kaposi sarcoma and implications of EphrinB2 blockade. Blood. 2009;113(1):254-263.

194. Hahn AS, et al. The ephrin receptor tyrosine kinase A2 is a cellular receptor for Kaposi's sarcoma-associated herpesvirus. Nat Med.
2012;18(6):961-966.

195. Chakraborty S, Veettil MV, Bottero V, Chandran B. Kaposi's sarcoma-associated herpesvirus interacts with EphrinA2 receptor to amplify signaling essential for productive infection. Proc Natl Acad Sci U S A. 2012;109(19):E1163-E1172.

196. Krown SE, Lee JY, Dittmer DP. AIDS Malignancy Consortium. More on HIV-associated Kaposi's sarcoma. N Engl JMed. 2008;358(5):535-536; author reply 536.

197. Maurer T, Ponte M, Leslie K. HIV-associated Kaposi's sarcoma with a high CD4 count and a low viral load. N Engl J Med. 2007;357(13):1352-1353.

198. Krown SE, et al. Stage-stratified approach to AIDS-related Kaposi's sarcoma: implications for resource-limited environments. JClin Oncol. 2014;32(23):2512-2513.

199. Bower M, et al. Prospective stage-stratified approach to AIDS-related Kaposi's sarcoma. JClin Oncol. 2014;32(5):409-414.

200.Webster-Cyriaque J, Edwards RH, Quinlivan EB, Patton L, Wohl D, Raab-Traub N. Epstein-Barr virus and human herpesvirus 8 prevalence in human immunodeficiency virus-associated oral mucosal lesions. JInfect Dis. 1997;175(6):1324-1332. 